\title{
AN EXTENSION OF A RESULT BY TALALYAN ON THE REPRESENTATION OF MEASURABLE FUNCTIONS BY SCHAUDER BASES
}

\author{
BEN-AMI BRAUN
}

\begin{abstract}
We define a class of Banach spaces which in particular include the $L^{p}, 1 \leqq p<\infty$, and the separable Orlicz spaces. We show that an arbitrary measurable function can be represented by a series of any Schauder basis chosen from that class of Banach spaces.
\end{abstract}

1. A classical question posed by Lusin [2] in 1915 asks whether for every measurable function $f$ on $[0,2 \pi]$, finite or infinite, there is a trigonometric series, with coefficients converging to zero, which converges almost everywhere to $f$.

It was only in 1937 that this problem was solved in the affirmative by Menshov [3] for the case where $f$ is finite almost everywhere.

By substituting convergence in measure for almost everywhere convergence, Menshov [4] then answered Lusin's question. He showed that for every measurable function on $[0,2 \pi]$, finite or infinite, there exists a trigonometric series with coefficients converging to zero which converges in measure to the function. Talalyan [5] proved Menshov's theorem for every normal Schauder basis in $L^{p}[a, b], p>1$.

This paper generalizes Talalyan's result to normalized Schauder bases chosen from a larger class of spaces which in particular includes $L^{1}[a, b]$ and the separable Orlicz spaces. We should note that the convergence used by Talalyan and in this paper is stronger than convergence in measure.

2. Let $L(E)$ be a Banach space of measurable functions on a measurable set $E \subset[0,1]$ with natural linear operations. As usual, identify functions equal almost everywhere. Postulate the following on $L(E)$ :

(2.1) $L(E)$ is contained in $L^{1}(E)$;

(2.2) $L(E)$ contains the function 1 ;

(2.3) If $f \in L(E)$, and if for a measurable function $g, 0 \leqq g(x) \leqq f(x)$ almost everywhere, then $g \in L(E)$;

(2.4) If $f \in L(E)$ and $\chi_{A}$ is the characteristic function of the measurable

Received by the editors October 22, 1970.

AMS 1970 subject classifications. Primary 46B15, 46E30; Secondary 41A30.

Key words and phrases. Schauder basis, representation of measurable functions, convergence in measure, asymptotic convergence.

(c) American Mathematical Society 1972 
set $A$, then $\left\|f \chi_{A}\right\| \equiv\|f\|_{A}$ goes to zero as $|A|$ goes to zero, where $|A|$ denotes the Lebesgue measure of $A$.

Next we define the mode of convergence, which is stronger than mere convergence in measure. We will say that a sequence $\left\{f_{n}\right\}$ of measurable, finite almost everywhere functions converges to the measurable function $f$ asymptotically in $L(E)$, if there are defined on $E$ two sequences $\left\{g_{n}\right\}$ and $\left\{\alpha_{n}\right\}$ of measurable, finite almost everywhere functions having the following properties:

(i) $f_{n}(x)=g_{n}(x)+\alpha_{n}(x)$, almost everywhere on $E$;

(ii) $\lim _{n \rightarrow \infty} g_{n}(x)=f(x)$, almost everywhere on $E$;

(iii) For any $\varepsilon>0$, there exists a measurable set $G \subset E$ such that $|G|>$ $1-\varepsilon$ and $\left\|\alpha_{n}\right\|_{G \rightarrow 0}$ as $n \rightarrow \infty$.

The following is the promised generalization of the representation theorem proved by Talalyan [5], [6].

MAIN THEOREM. Let $\left\{\phi_{n}\right\}_{n=1}^{\infty}$ be an arbitrary normalized basis of the space $L(E)$. Then for any measurable function $f$ defined on $E$, which may be infinite on a set of positive measure, there exists a series

$$
\sum_{n=1}^{\infty} b_{n} \phi_{n} \text { with } \lim _{n \rightarrow \infty} b_{n}=0
$$

which converges to $f$ asymptotically in the norm of $L(E)$.

3. Before proceeding with the proof we need to state some properties of $L(E)$. They follow immediately from

THeOREM (LoRentz). Suppose that $X$ satisfies (2.2)-(2.4) and $Y$ consists of all measurable functions $g$ defined on E for which $\int_{E} f(x) g(x) d x$ exists for all $f$ in $X$. Then

$$
(f, g)=\int_{E} f(x) g(x) d x, \quad g \in Y,
$$

is the general form of a continuous linear functional on $X$, and its norm is equal to $\|g\|^{*}=\sup _{\|f\| \leqq 1}(f, g)<\infty$.

COROLlary 3.1. We have an Hölder inequality. If $f \in L(E)$ and $g \in$ $L^{*}(E)$ then $|(f, g)| \leqq\|f\|\|g\|^{*}$.

Corollary 3.2. If $g \in L^{*}(E)$ then $g \in L^{1}(E)$.

Corollary 3.3. If $f \in L(E)$, then $\|f\|=\sup _{\|g\|^{*} \leqq 1}(f, g), g \in L^{*}(E)$.

COROLlaRY 3.4. If $f \in L(E)$ and if $|g(x)| \leqq|f(x)|$ almost everywhere, then $\|g\| \leqq\|f\|$. 
COROLlary 3.5. Under the hypotheses of the theorem, $L^{*}(E)$ satisfies postulates (2.2) and (2.3).

COROLlaRY 3.6. $L^{*}(E)$ contains the characteristic functions of all measurable subsets of $E$.

COROllaRY 3.7. If $g \in L^{*}(E)$ and $|f(x)| \leqq|g(x)|$ almost everywhere, then $\|f\|^{*} \leqq\|g\|^{*}$.

COROLLARY 3.8. Uniform convergence implies convergence in the norm both in $L(E)$ and in $L^{*}(E)$.

4. Now we are ready to state three lemmas, which are restatements of Talalyan's lemmas [5] in a more general setting. We shall prove only the first lemma since the other two do not involve the conjugate space and Talalyan's proofs apply, also the proof of the Main Theorem is the same as that of its predecessor [6].

LEMMA 4.1. Let $\phi$ be a bounded measurable function on $\Delta \equiv[0,1]$ such that $\phi(x)=0$, if $x \notin E_{0} \subset \Delta$. Let $\psi_{1}, \psi_{2}, \cdots, \psi_{n}$ belong to $L^{*}(\Delta)$. Then given $\varepsilon>0$ and $0<\varepsilon_{0}<1$ there exists a bounded measurable function $f$ and $a$ set $e \subset E_{0}$ such that:

(a) $f(x)=0$, if $x \notin e$ and $|e| \leqq \varepsilon_{0}|\Delta|$;

(b) $\|f\| \leqq\left(M / \varepsilon_{0}\right)\left\|\chi_{\Delta}\right\|$, where $M=\sup _{x \in \Delta} \phi(x)$;

(c) $\left|\int_{\Delta}(\phi-f) \psi_{k}\right|<\varepsilon, 1 \leqq k \leqq n$.

LEMMA 4.2. Let $\left\{\phi_{n}\right\}_{n=1}^{\infty}$ be a normalized basis for $L(E)$. Let $f$ be a bounded measurable function such that $f(x)=0$ when $x \notin E_{0} \subset E$. Then, given $\varepsilon>0$, there exists a function $F \in L(E)$ and a measurable set $e_{0}$ such that:

( $\alpha) F(x)=f(x)$ when $x \notin e_{0}, e_{0} \subset E_{0}$ and $\left|e_{0}\right|<\varepsilon$;

( $\beta)\left|a_{n}\right|<\varepsilon$, for all $n$, where the $a_{n}$ are the coefficients of the expansion of $F$;

$(\gamma)$ for all measurable sets $e \subset E \backslash e_{0}$,

$$
\left\|\sum_{k=1}^{n} a_{k} \phi_{k}\right\|_{e} \leqq \varepsilon+\|f\|_{e} \text { for all } n .
$$

Notice that the function $\phi$ of Lemma 4.1 and $f$ of Lemma 4.2 are taken to be bounded while Talalyan lets them be an arbitrary function of $L^{p}, p>1$. This does not affect the third lemma.

LEMMA 4.3. Let $\left\{\phi_{n}\right\}_{n=1}^{\infty}$ be a normal basis for $L(E)$. Let $g$ be a measurable function finite almost everywhere on $E$. Then given $\varepsilon>0$ and a positive integer $N$, there exists a measurable set $e_{0}$ and real numbers $b_{N+1}, \cdots, b_{m}$ 
such that:

(1) $e_{0} \subset E$ and $\left|e_{0}\right|<\varepsilon$;

(2) $\left|b_{k}\right|<\varepsilon, N+1 \leqq k \leqq m$;

(3) $\left\|\sum_{k=\mathrm{N}+1}^{m} b_{k} \phi_{k}-g\right\|_{\left(E \backslash e_{0}\right)}<\varepsilon$;

(4) $\left\|\sum_{k=N+1}^{s} b_{k} \phi_{k}\right\|_{e} \leqq \varepsilon+\|g\|_{e}$, where $N+1 \leqq s \leqq m$ and $e$ is an arbitrary measurable subset of $E \backslash e_{0}$.

Proof of Lemma 4.1. If $\phi(x)=0$ almost everywhere, put $f(x)=0$, and we are finished. So, assume that $\phi(x) \neq 0$ on a set of positive measure, and let $\psi$ be an arbitrary continuous function.

We divide $\Delta$ into $n$ equal subintervals $\Delta_{1}, \Delta_{2}, \cdots, \Delta_{n}$, and define the following functions:

$$
\begin{array}{cl}
\phi^{n}(x)=\frac{1}{\left|\Delta_{k}\right|} \int_{\Delta_{k}} \phi & \left(x \in \Delta_{k}, k=1,2, \cdots, n\right) ; \\
\psi^{n}(x)=\frac{1}{\left|\Delta_{k}\right|} \int_{\Delta_{k}} \psi & \left(x \in \Delta_{k}, k=1,2, \cdots, n\right) .
\end{array}
$$

Let the functions take the value zero at the points of division.

It is easy to see that $\phi^{n}(x) \rightarrow \phi(x)$ almost everywhere on $\Delta$. Therefore, for a given $\varepsilon>0$, there exists a set $G \subset[0,1]$ such that $|G|>1-\varepsilon$ and $\phi^{n}(x) \rightarrow \phi(x)$ uniformly on $G$. Hence, by Corollary 2.8 ,

$$
\left\|\left[\phi^{n}-\phi\right] \chi_{G}\right\| \rightarrow 0 \text { as } n \rightarrow \infty .
$$

Since $\phi$ is bounded, say by $M$, we have $|\phi(x)|<M$ and $\left|\phi^{n}(x)\right|<M$. From this, it follows that $\left|\phi^{n}-\phi\right| \leqq\left|\phi^{n}-\phi\right| \chi_{G}+2 M \chi_{(\Delta \mid G)}$, and hence

$$
\left\|\phi^{n}-\phi\right\| \leqq\left\|\phi^{n}-\phi\right\|_{G}+2 M\|1\|_{(\Delta \backslash G)} .
$$

Now given $\delta>0$, we can find an $\varepsilon>0$, small enough so that the measurable set $G$ has the property that $\|1\|_{(\Delta ; G)}<\delta(4 M)^{-1}$. By virtue of (4.1), (4.2) and the choice of the set $G$, we obtain $\left\|\phi^{n}-\phi\right\|<\delta$, for sufficiently large $n$. Since $\delta$ was arbitrary we have

$$
\left\|\phi^{n}-\phi\right\| \rightarrow 0 \text { as } n \rightarrow \infty .
$$

From the continuity of $\psi$ we get the uniform convergence of $\psi^{n}$ to $\psi$ and from Corollary 2.8 we obtain

$$
\left\|\psi^{n}-\psi\right\|^{*} \rightarrow 0 \quad \text { as } n \rightarrow \infty .
$$

Next, using the Hölder inequality for $L(\Delta)$ coupled with (4.3), (4.4) and the fact that the $\left\|\psi^{n}\right\|^{*}$ are uniformly bounded we obtain

$$
\lim _{n \rightarrow \infty}\left|\int_{\Delta} \psi^{n}\left[\phi^{n}-\phi\right]+\int_{\Delta} \phi\left[\psi^{n}-\psi\right]\right|=0 .
$$


444

B.-A. BRAUN

[August

The above together with the relation

$$
\phi^{n}(x) \psi^{n}(x)-\phi(x) \psi(x)=\psi^{n}(x)\left[\phi^{n}(x)-\phi(x)\right]+\phi(x)\left[\psi^{n}(x)-\psi(x)\right]
$$

leads to

$$
\lim _{n \rightarrow \infty} \int_{\Delta} \phi^{n} \psi^{n}=\int_{\Delta} \phi \psi
$$

Define functions $f_{n}(n=1,2,3, \cdots)$ as follows. In each interval $\Delta_{k}$ choose as interval $\delta_{k}$ of length $\left|\delta_{k}\right|=\varepsilon_{0}\left|\Delta_{k}\right|$, and let

$$
\begin{aligned}
f_{n}(x) & =\frac{1}{\varepsilon_{0}\left|\Delta_{k}\right|} \int_{\Delta_{k}} \phi, & & x \in \delta_{k}(k=1,2, \cdots, n) ; \\
& =0, & & x \notin \bigcup_{k=1}^{n} \delta_{k} .
\end{aligned}
$$

Now

$$
\begin{aligned}
\int_{\Delta} f_{n} \psi^{n} & =\sum_{k=1}^{n} \int_{\delta_{k}} f_{n} \psi^{n}=\sum_{k=1}^{n} \int_{\delta_{k}}\left[\left(\frac{1}{\varepsilon_{0}\left|\Delta_{k}\right|} \int_{\Delta_{k}} \phi\right)\left(\frac{1}{\left|\Delta_{k}\right|} \int_{\Delta_{k}} \psi^{n}\right)\right] \\
& =\sum_{k=1}^{n} \frac{1}{\left|\Delta_{k}\right|}\left(\int_{\Delta_{k}} \phi\right)\left(\int_{\Delta_{k}} \psi^{n}\right)=\sum_{k=1}^{n} \int_{\Delta_{k}}\left(\frac{1}{\left|\Delta_{k}\right|} \int_{\Delta_{k}} \phi\right) \psi^{n} \\
& =\sum_{k=1}^{n} \int_{\Delta_{k}} \phi^{n} \psi^{n}=\int_{\Delta} \phi^{n} \psi^{n} .
\end{aligned}
$$

Thus, we have

$$
\int_{\Delta} f_{n} \psi^{n}=\int_{\Delta} \phi^{n} \psi^{n}
$$

By virtue of (4.5) and (4.6), we obtain

$$
\lim _{n \rightarrow \infty} \int_{\Delta} f_{n} \psi^{n}=\int_{\Delta} \phi \psi
$$

On the other hand hence

$$
\lim _{n \rightarrow \infty}\left|\int_{\Delta} f_{n} \psi^{n}-\int_{\Delta} f_{n} \psi\right|=0
$$

$$
\lim _{n \rightarrow \infty} \int_{\Delta} f_{n} \psi=\int_{\Delta} \phi \psi
$$

Proceed by defining the following functions

$$
\begin{aligned}
g_{n}(x) & =f_{n}(x), & & x \in E_{0} \cap \delta_{k} ; \\
& =0, & & x \in E_{0} \cap\left(\bigcup_{k=1}^{n} \delta_{k}\right) .
\end{aligned}
$$


By virtue of (4.3) and the support of $\phi$ we obtain

$$
\left\|\phi^{n}\right\|_{\left(\Delta \backslash E_{0}\right)}=\left\|\phi^{n}-\phi\right\|_{\left(\Delta \backslash E_{0}\right)} \leqq\left\|\phi^{n}-\phi\right\| \rightarrow 0 \text { as } n \rightarrow \infty .
$$

It follows immediately that

$$
\left\|f_{n}-g_{n}\right\|=\left\|f_{n}\right\|_{\left(\Delta \backslash E_{0}\right)} \leqq \frac{1}{\varepsilon_{0}}\left\|\phi^{n}\right\|_{\left(\Delta \mid E_{0}\right)} \rightarrow 0 \text { as } n \rightarrow \infty .
$$

In view of the above it is readily seen that

$$
\left|\int_{\Delta} g_{n} \psi-\int_{\Delta} f_{n} \psi\right| \rightarrow 0 \text { as } n \rightarrow \infty
$$

this together with (4.7) gives

$$
\lim _{n \rightarrow \infty} \int_{\Delta} g_{n} \psi=\int_{\Delta} \phi \psi
$$

The next step is to prove (4.8) with $\psi$ bounded, say by $K$, and not necessarily continuous. By Lusin's theorem, we can find functions $\phi_{i}$, $i=1,2,3, \cdots$, which are continuous on $\Delta$, bounded by $K$ and such that measure $\left[\phi_{i} \neq \psi\right]<2^{-i}$. Now

$$
\begin{aligned}
\left|\int_{\Delta}\left(g_{n}-\phi\right) \psi\right| & <\left|\int_{\Delta}\left(g_{n}-\phi\right)\left(\psi-\phi_{i}\right)\right|+\left|\int_{\Delta}\left(g_{n}-\phi\right) \phi_{i}\right| \\
& =\left|\int_{\left[\phi_{i} \neq \psi\right]}\left(g_{n}-\phi\right)\left(\psi-\phi_{i}\right)\right|+\left|\int_{\Delta}\left(g_{n}-\phi\right) \phi_{i}\right| \\
& \leqq\left\|g_{n}-\phi\right\|_{\left[\phi_{i} \neq \psi\right]}\left\|\psi-\phi_{i}\right\|^{*}+\left|\int_{\Delta}\left(g_{n}-\phi\right) \phi_{i}\right| \\
& \leqq\left(M / \varepsilon_{0}+M\right)\|1\|_{\left[\phi_{i} \neq \psi\right]} 2 K\|1\|^{*}+\left|\int_{\Delta}\left(g_{n}-\phi\right) \phi_{i}\right| .
\end{aligned}
$$

Now choose $i_{0}$ large enough so that

$$
\|1\|_{\left[\phi_{i} \neq \psi\right]} \leqq \varepsilon_{0}\left(M+M \varepsilon_{0}\right)^{-1}\left(4 K\|1\|^{*}\right)^{-1} \varepsilon .
$$

By virtue of (4.8) we can choose $N$ large enough so that

$$
\left|\int\left(g_{n}-\phi\right) \phi_{i_{0}}\right|<\varepsilon / 2, \quad n \geqq N .
$$

Putting the pieces together, we obtain (4.8) for the case where $\psi$ is bounded.

Last step: given $\psi \in L^{*}(\Delta)$, choose functions $\phi_{i}, i=1,2,3, \cdots$, which are bounded on $\Delta$ with measure $\left[\phi_{i} \neq \psi\right]<2^{-i}$ and $\phi_{i}(x)=0$, when $x \in$ $\left[\phi_{i} \neq \psi\right]$. Now

$$
\left|\int_{G}\left(g_{n}-\phi\right) \psi\right| \leqq\left\|g_{n}-\phi\right\|_{G}\|\psi\|^{*} \leqq M\left(\varepsilon_{0}^{-1}+1\right)\|1\|_{G}\|\psi\|^{*}
$$


Hence, given $\varepsilon>0$ there exists $\delta>0$ such that $|G|<\delta$ implies that the left-hand side of (4.9) is less than $\varepsilon$. Next,

$$
\left|\int_{\Delta}\left(g_{n}-\phi\right) \psi\right| \leqq\left|\int_{\left[\phi_{i}=\psi\right]}\left(g_{n}-\phi\right) \phi_{i}\right|+\left|\int_{\left[\phi_{i} \neq \psi\right]}\left(g_{n}-\phi\right) \psi\right| \text {. }
$$

Choose $i_{0}$ so that $2^{-i}<\delta$. Then we have measure $\left[\phi_{i_{0}} \neq \psi\right]<\delta$, and hence the second expression on the right-hand side of (4.10) is less than $\varepsilon$. Now, since

$$
\left|\int_{\left[\phi_{i_{0}}=\psi\right]}\left(g_{n}-\phi\right) \phi_{i_{0}}\right|=\left|\int_{\Delta}\left(g_{n}-\phi\right) \phi_{i_{0}} \chi_{\left[\phi_{i_{0}}=\psi\right]}\right|,
$$

we may apply (4.8) and choose a natural number $N$ such that the first expression on the right-hand side of (4.10) is less than $\varepsilon$, for all $n>N$. Therefore for any given $\varepsilon>0$ there exists $N(\varepsilon)$ such that $n>N(\varepsilon)$ implies that the left-hand side of $(4.10)$ is less than $2 \varepsilon$. Hence, we have

$$
\lim _{n \rightarrow \infty} \int_{\Delta} g_{n} \psi=\int_{\Delta} \phi \psi, \text { for all } \psi \in L^{*}(\Delta) \text {. }
$$

Let $\varepsilon>0$ be given. Then by $(4.11)$, we can choose $N=N(\varepsilon, n)$ to insure that

$$
\begin{aligned}
& \left|\int_{\Delta} g_{n} \psi_{i}-\int_{\Delta} \phi \psi_{i}\right|<\varepsilon, \quad i=1,2, \cdots, n, \\
& \text { where } \psi_{i} \in L^{*}(\Delta), i=1,2, \cdots, n \text {. }
\end{aligned}
$$

Then the bounded function $f=g_{N}$ and the set $e=E_{0} \cap\left(\bigcup_{k=1}^{N} \delta_{k}\right)$ satisfy the lemma.

\section{BIBLIOGRAPHY}

1. G. G. Lorentz, Bernstein polynomials, Math. Expositions, no. 8, Univ. of Toronto Press, Toronto, 1953. MR 15, 217.

2. N. Luzin, Integral and trigonometric series, GITTL, Moscow, 1951, p. 236.(Russian) MR 14, 2.

3. D. E. Men'šov, Sur la représentation des fonctions mesurables par des séries trigonométriques, Mat. Sb. 9 (51) (1941), 667-692. MR 3, 106.

4. - On convergence in measure of trigonometric series, Trudy Mat. Inst. Steklov. 32 (1950); English transl., Amer. Math. Soc. Transl. (1) 3 (1962), 196-270. MR 12, 254.

5. A. A. Talaljan, Representation of measurable functions by series, Uspehi Mat. Nauk 15 (1960), no. 5 (95), 77-141=Russian Math. Surveys 15 (1960), no. 5, 75-136. MR 23 \#A2704.

6. - , Limit functions of series in bases of the space $L^{p}$, Mat. Sb. 56 (98) (1962), 353-374; English transl., Amer. Math. Soc. Transl. (2) 43 (1964), 51-74. MR 27 \#1767.

Department of Mathematics, University of South Florida, Tampa, Florida 33620 